\title{
Relating pitch awareness to phonemic awareness in children: implications for tone-deafness and dyslexia
}

\author{
Psyche Loui ${ }^{*}$, Kenneth Kroog ${ }^{2}$, Jennifer Zuk', Ellen Winner ${ }^{2,3}$ and Gottfried Schlaug ${ }^{1}$ \\ ' Department of Neurology, Beth Israel Deaconess Medical Center, Harvard Medical School, Boston, MA, USA \\ 2 Department of Psychology, Boston College, Chestnut Hill, MA, USA \\ 3 Project Zero, Harvard Graduate School of Education, Cambridge, MA, USA
}

Edited by:

Lutz Jäncke, University of Zurich,

Switzerland

Reviewed by:

Lutz Jäncke, University of Zurich, Switzerland

Nina Kraus, Northwestern University, USA

Franziska Degé, Justus-Liebig-

University, Germany

*Correspondence:

Psyche Loui, Department of Neurology, Beth Israel Deaconess Medical Center, Harvard Medical School, 330 Brookline Avenue, Palmer 127, Boston, MA 02215, USA.

e-mail: ploui@bidmc.harvard.edu
Language and music are complex cognitive and neural functions that rely on awareness of one's own sound productions. Information on the awareness of vocal pitch, and its relation to phonemic awareness which is crucial for learning to read, will be important for understanding the relationship between tone-deafness and developmental language disorders such as dyslexia. Here we show that phonemic awareness skills are positively correlated with pitch perception-production skills in children. Children between the ages of seven and nine were tested on pitch perception and production, phonemic awareness, and IQ. Results showed a significant positive correlation between pitch perception-production and phonemic awareness, suggesting that the relationship between musical and linguistic sound processing is intimately linked to awareness at the level of pitch and phonemes. Since tone-deafness is a pitch-related impairment and dyslexia is a deficit of phonemic awareness, we suggest that dyslexia and tone-deafness may have a shared and/or common neural basis.

Keywords: music, language, development, phonemic awareness, pitch perception, production, tone-deafness, dyslexia

\section{INTRODUCTION}

Developmental dyslexia is a disorder in which children with normal intelligence and sensory abilities show learning deficits for reading (Démonet et al., 2004). Children with dyslexia are characterized by an impairment in phonemic awareness, which is the ability to process and manipulate spoken words made up of individual sounds or phonemes (Bradley and Bryant, 1983; Stanovich, 1988; Stahl and Murray, 1994; Snow et al., 1998). In recent years, the relationship between phonemic awareness and musical ability is a topic of increased interest. Studies have demonstrated significant relationships between phonemic awareness and musical sound processing in children as well as adults (Stadler, 1990; Anvari et al., 2002; Jentschke et al., 2005; Jones et al., 2009a; Moreno et al., 2009). One line of research shows that the processing of rhythm and meter in music predicts phonological difficulties in children with dyslexia, suggesting that dyslexia may arise from a difficulty in processing the fast temporal structure of speech sounds (Tallal and Piercy, 1973; Merzenich et al., 1996; Overy, 2003; Benasich et al., 2006; Huss et al., 2011). Since consonants within a language are largely differentiated by temporally rapid sound elements, the ability to process temporally rapid changes is important for phonemic awareness. Support for this theory comes from the finding that auditory training that involves the gradual increase in temporal frequency of tones leads to improvements in auditory processing speed, speech discrimination, and language comprehension (Tallal, 2004; Tallal and Gaab, 2006).

Another line of research has shown links between phonemic awareness and pitch processing, rather than temporal processing, of musical sounds. As different consonants and vowels in speech are characterized by different frequency as well as temporal content, and pitch is the direct perceptual correlate of frequency, pitch perception and phoneme perception might share the same process of frequency discrimination. Thus, sensitivity to frequency changes may be important for reading ability as well. In previous research, phonemic awareness was shown to be correlated with pitch discrimination, pitch awareness (as assessed using the tonal test of the Gordon Primary Measures of Audiation (PMMA; Gordon, 1979), melody discrimination, and tone manipulation tasks (Lamb and Gregory, 1993; Anvari, et al., 2002; Peynircioglu et al., 2002; Bolduc, 2005; Forgeard et al., 2008a). Additionally, children with specific language impairment were shown to be impaired at pitch discrimination tasks, further suggesting that the link between phonemic awareness and musical ability holds in the domain of pitch (Mengler et al., 2005).

Individuals who are tone-deaf also have impaired pitch processing. Tone-deafness, or congenital amusia, is a deficit of musical ability that according to some reports affects up to $17 \%$ of the normal adult population (Kalmus and Fry, 1980; Cuddy et al., 2005; Loui et al., 2009; Peretz et al., 2009). Little is known regarding the developmental trajectory of tone-deafness in children, although the topic is beginning to be explored (Lebrun et al., in press). Adults who are tone-deaf have deficits in pitch production as well as perception, and show a mismatch between their pitch perception and production abilities (Loui et al., 2008). They also show impairments in phonemic awareness (Jones et al. 2009a) as well as impairments in the processing of speech intonation (Liu et al., 2010) and in the understanding of lexical tones in a tonal language (Nan et al., 2010). Taken together, the research suggests a relationship between phonemic awareness and pitch perception. However, the relationship between phonemic awareness and pitch production is yet unclear, especially in young children. Understanding tone-deaf behavior in children - as characterized by deficits in 
pitch perception, production, and the relationship between the two - and the possible relationship between tone-deafness and phonemic awareness, is important for characterizing the specificity of difficulties that children with developmental language disorders might face in their auditory environment.

In what follows, we examine the relationship between pitch perception and production, on the one hand, and phonemic awareness, on the other hand, in children between the ages of seven and nine. This age range was chosen because it is the earliest time when measurable deficits in literacy development can be readily identified (Shaywitz and Shaywitz, 2005). We hypothesized that the degree of correlation between perception and production performance (which we call pitch awareness) should be positively associated with level of phonemic awareness.

\section{MATERIALS AND METHODS PARTICIPANTS}

Thirty-two children (16 females, 16 males) were recruited from Grant School No. 7 in Passaic, NJ, USA, and from schools in the greater Boston area. Children's mean age was 7.6 years ( $\mathrm{SD}=0.7$ years). Children had a mean of 0.4 years of musical training outside of normal school education (range $=0-3$ years, with five children having more than a year of musical training). Median household income was between US \$35,000 and \$50,000.

\section{STIMULI AND PROCEDURE}

Prior to testing, parents were contacted to obtain informed consent and to fill out a basic questionnaire. This questionnaire included items asking for the family's contact information as well as the child's date of birth and whether or not they had received any musical training outside of normal schooling. Each child was tested on a battery of tests that assessed phonemic awareness, pitch perception and production, and general intelligence. The pitch perception and production tests were used previously in our lab with tone-deaf adults (Loui, et al., 2008). Phonemic awareness scores were obtained from a combination of existing tests, the Sound Categorization tests (Bradley and Bryant, 1985) and Auditory Analysis tests (Rosner and Simon, 1971).

\section{Phonemic awareness tests}

Two phonemic awareness tests were administered: the Sound Categorization Test (Bradley and Bryant, 1985) and the Auditory Analysis Test (Rosner and Simon, 1971). The Sound Categorization Test presents participants with four words per trial, with the instruction to find the word with the last sound that differed (e.g., "fan cat hat mat" - target answer: "fan"), the first sound that differed (e.g., "rot rod rock box" - target answer: "box"), and the middle sound that differed (e.g., "mop hop tap lop" - target answer: "tap"). Two practice trials followed by 10 test trials were administered in each category (last sound, first sound, middle sound), yielding a total of 36 trials (six practice and 30 test).

The Auditory Analysis Test presents participants with two practice items followed by 40 test items. Each item consists of a compound word (e.g., "cowboy") with instructions to say the word without one of the compounds (e.g., "say cowboy without boy." Target answer: "cow").

\section{Pitch perception and production test}

The Pitch Perception and Production Test was created in our lab and reveals mismatched pitch perception and production abilities in the tone-deaf adults (Loui, et al., 2008): while non-tone-deaf controls demonstrate similar levels of performance between pitch perception and production in this test, tone-deaf individuals show varied levels of performance, suggesting that processing streams for perception and production might be dissociated in the auditory system. The test presents participants with pairs of pure tones forming intervals. Within each pair, the first tone has a fundamental frequency of $500 \mathrm{~Hz}$ and the second tone ranges in frequency from $267 \mathrm{~Hz}$ to $750 \mathrm{~Hz}$. Each tone was $500 \mathrm{~ms}$ in duration, with a gap of $500 \mathrm{~ms}$ between the two tones. Thirteen tone pairs were presented. After each presentation, the tasks were first to reproduce the tone pair by humming (pitch production test), and then to state whether the second tone was higher or lower than the first (pitch perception test). The order of the two tasks was counterbalanced across participants.

\section{Non-verbal intelligence test}

Non-verbal intelligence was assessed using the Kaufman brief intelligence test (KBIT; Kaufman and Kaufman, 1990). This consisted of a series of up to 40 test items. Each test item consists of a puzzle matrix with a single missing piece. Children were asked to choose the item that fit best within the matrix given a choice of several puzzle pieces. The KBIT is a well-established test that has been shown to be correlated with non-verbal intelligence in children of the age of four and above (Kaufman and Kaufman, 1990). This test was administered so that we could control for possible confounds of non-verbal intelligence.

\section{DATA ANALYSIS}

Age (years and months) at the time of testing was calculated from date of birth as reported by the parents. Socio-economic status of each child was estimated based on the median household income reported in US census data (http://2010.census.gov/2010census/ data/) for each child's zip code.

On average, children scored 22 correct out of 40 (74\%) on the Sound Categorization test $(\mathrm{SD}=22 \%)$ and 21 correct out of $40(54 \%)$ on the Auditory Analysis test (SD = 27\%). Since there was no a priori reason to expect that performance on Sound Categorization and Auditory Analysis tests might show different relationships with pitch perception and production, each child's raw scores (not age-normalized) for Sound Categorization and Auditory Analysis tests were summed to form a single Phonemic Awareness score for statistical comparisons against pitch-awareness measures.

Previous studies have found that the agreement between pitch perception and production is low among adults who are tone-deaf (Loui, et al., 2008). To obtain an unbiased measure of internal consistency of children's perception and production skills, we calculated a single Pitch Perception-Production index for each child using the following procedure. For each trial, the direction of each participant's hummed pitch intervals (i.e., the production task) was first coded as +1 (for rising intervals) or -1 (for falling intervals). Then the verbal response in the perception task was coded for 
each trial with $a+1$ (if they said that the second tone was higher than the first) or $a-1$ (if they said that the second tone was lower than the first). The raw scores for the production and perception tasks were then correlated to form the Perception-Production index for each participant. A correlation score of 1.0 indicates perfect congruence between the perception and production of interval directions (i.e., the child always produced an ascending interval in the trials where s/he reported perceiving an ascending interval), a score of 0 indicated no correlation between perception and production (i.e., on trials where the child produced an ascending interval, s/he was equally likely to report hearing an ascending or a descending interval), and a correlation of -1.0 indicates opposite performance between perception and production. This correlation was then Fisher-transformed to obtain a $z$-score for each subject. The Pitch Perception-Production Index indicated whether the participants hummed back the same pitch directions that they perceived from the recordings. This score does not reflect whether each of the perception/production responses was correct, but only whether there was internal consistency between each child's perception and production.

Bivariate pairwise correlations were then performed to evaluate relationships between each pair of variables (Phonemic Awareness score, Pitch Perception-Production index, KBIT score, and Age). In addition, in order to eliminate the possible influences of confounding variables of IQ and age, partial correlations were run between Phonemic Awareness scores and the Pitch Perception-Production index, while controlling for KBIT, age, SES, and musical training.

\section{RESULTS}

Pairwise correlations revealed five significant positive correlations: Pitch Perception-Production was correlated with Phonemic Awareness $(r=0.795, p<0.001)$, KBIT was correlated with Phonemic Awareness $(r=0.593, p=0.001)$, Pitch Perception-Production was correlated with KBIT $(r=0.708, p<0.001)$, Pitch PerceptionProduction was correlated with Age $(r=0.576, p=0.003)$, and KBIT was correlated with Age $(r=0.695, p<0.001)$, as shown in Table 1 .

Having established multiple significant bivariate relationships between each set of variables, the next step was to determine whether the relationship between Phonemic Awareness and Pitch Perception-Production existed even after controlling for general intelligence (as measured by the KBIT), age, SES, and musical training. A partial correlation was run examining the relationship of the Perception-Production Index and Phonemic Awareness, with age,
KBIT, SES, and number of years of musical training controlled. Partial correlations revealed significant correlations between Pitch Perception-Production and Phonemic Awareness even after partialing out the effects of intelligence, age, SES, and musical training $\left(r_{\text {partial }}=0.586, p=0.008\right)$. The relationship between Phonemic Awareness and Pitch Perception-Production is shown in Figure 1.

Considering the two separate components of the Pitch Perception-Production index, pitch perception-only and production-only scores were not significantly correlated with the phonemic awareness score (correlation between phonemic awareness and perception scores: $r=0.29, p=0.17$; between phonemic awareness and production scores: $r=0.062, p=0.80)$. Perception-only and production-only scores were also not significantly correlated with the Sound Categorization and Auditory Analysis tests that made up the Phonemic Awareness score (correlation between Sound Categorization and pitch perception: $r=0.15, p=0.47$; between Sound Categorization and pitch production: $r=0.11, p=0.60$; between Auditory Analysis and pitch perception: $r=0.19, p=0.40$; between Auditory Analysis and pitch production: $r=-0.077$, $p=0.72$ ). However, the Pitch Perception-Production index was significantly correlated with each of the two Phonemic Awareness tests (correlation between Perception-Production index and Sound Categorization: $r=0.671, p<0.001$; between Perception-Production

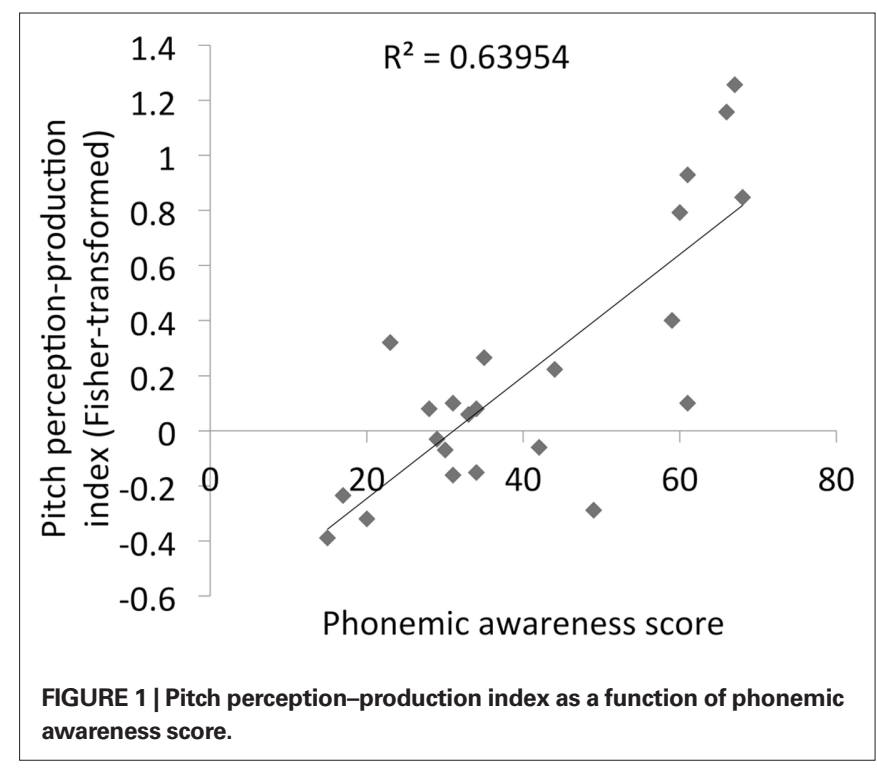

Table 1 | Pairwise correlation coefficients between variables of phonemic awareness scores, pitch perception-production scores, Kaufman brief intelligence test scores, age, musical training, and SES.

\begin{tabular}{|c|c|c|c|c|c|}
\hline & Pitch perception-production & KBIT & Age & Musical training & SES \\
\hline Phonemic awareness & $0.800 * *$ & $0.593^{* *}$ & 0.347 & $0.582^{* *}$ & $0.741^{* *}$ \\
\hline Pitch perception-production & & $0.735^{* *}$ & $0.576^{* *}$ & $0.802^{* *}$ & $0.489 * *$ \\
\hline $\mathrm{KBIT}$ & & & $0.695^{* *}$ & $0.901 * *$ & $0.742^{* *}$ \\
\hline Age & & & & $0.755^{* *}$ & $0.641^{* *}$ \\
\hline Musical training & & & & & $0.805^{* *}$ \\
\hline
\end{tabular}


index and Auditory Analysis test: $r=0.791, p<0.001$ ), suggesting that it is the combination of pitch perception and production skills, rather than each of the two skills considered separately, that accounts for individual differences in phonemic awareness.

\section{DISCUSSION}

This study demonstrates that phonemic awareness is correlated with pitch awareness, as assessed by the degree of agreement between pitch perception and pitch production. These results are independent of age, non-verbal IQ, SES, and musical training. While prior studies have shown that phonemic awareness, a crucial predictor of reading ability, is related to musical ability (Anvari, et al., 2002; Forgeard et al., 2008b; Jones, et al., 2009a), our study is the first to demonstrate an association between phonemic awareness and level of agreement between pitch perception and pitch production. Given that individuals with dyslexia are impaired in phonemic awareness (Stahl and Murray, 1994) and tone-deaf individuals show mismatches between pitch perception and production (Loui, et al., 2008), the current results suggest an intriguing association between dyslexia and tone-deafness and warrant further investigation of a possible shared impairment across these disorders.

The present correlational findings now motivate research into whether phonemic processing and pitch processing are mediated by a shared neural substrate. It has been shown that tone-deafness in adults is characterized by a mismatch between pitch perception and production (Loui, et al., 2008; Liu, et al., 2010) as well as to deficits in phonemic awareness (Jones et al., 2009b). It has also been established that phonemic awareness is deficient in dyslexia (Snow, et al., 1998; Stanovich, 1988; Wood et al., 2005). Taken together, the present results suggest that tone-deafness and dyslexia may be intimately related. Since the task of perceiving and producing pitch intervals requires awareness of one's own voice, the present results suggest that a deficit in awareness of one's own voice may underlie both tone-deafness and dyslexia. Although deficits in pitch awareness are certainly not the sole deficit in dyslexia, a pitch-awareness deficit could be one of many risk factors for language impairment (Leppanen et al., 2002).

The relationship between pitch perception and pitch production has been investigated in previous studies involving auditory feedback of one's own voice in adults (Burnett and Larson, 2002; Pfordresher, 2005). While auditory feedback is a useful way to investigate how one responds to one's own voice, another measure of vocal awareness, especially in the domain of pitch, can be best assessed by recording subjects' perception and production of pitched intervals (pairs of pitches) and comparing each individual's perceptual response and production output for internal consistency. Our previous work has shown that tone-deaf adults lack conscious awareness of their own vocal productions of pitched intervals (Loui, et al., 2008). Adults identified as tone-deaf by the Montreal Battery for Evaluation of Amusia (Peretz et al., 2003) as well as by their responses to psychophysically defined frequency discrimination thresholds (Foxton et al., 2004) demonstrate a paradoxical mismatch between pitch interval perception and production, where they could produce small pitched intervals in the correct direction despite being unable to perceive the direction of the pitched interval according to their own verbal report. Although tone-deafness (or congenital amusia) has been described in adults for decades, the incidence of tone-deafness in children is unknown, with the first case report of amusia having only recently been reported in one child (Lebrun et al., in press). Patterns of behavior from some of the children in the present study, i.e., children who showed negative correlations between perception and production performance, are similar to the previously reported perception-production mismatch in tone-deaf adults. In that regard, the pitch perception-production tests in the current study might be useful in the future as a sensitive screening test for early identification of tone-deafness in childhood. While it is unclear whether this mismatch behavior is a sign of a developing auditory-motor system, or whether these children are currently tone-deaf, or will develop into tone-deaf adults, the present results show that they exhibit behavioral characteristics of tone-deaf adults and thus may put them into an at-risk category for tone-deafness.

Neuroimaging work has shown that the behavioral pattern of mismatch between pitch interval perception and production is associated with atypical white matter connectivity in the arcuate fasciculus, which connects the superior and middle temporal gyri in the temporal lobe with the posterior inferior frontal gyrus in the frontal lobe (Loui, et al., 2009). Notably, individuals who showed more congruent perception and production abilities had larger inferior branches of the right arcuate fasciculus. Given that arcuate fasciculus volume is correlated between the left and right hemispheres (Wahl et al., 2010) but also shows significant hemispheric asymmetry (Catani et al., 2005; Vernooij et al., 2007), the present results may be a result of structural correspondence between left and right arcuate fasciculi during development, such that children with a well-developed right inferior arcuate fasciculi will also have highly internally consistent pitch perception and production abilities and possess well-developed left inferior arcuate fasciculus, which may be involved in phonemic awareness. Alternatively, the two domains of sound awareness (pitch and phonemes) may involve the same neural structures, providing further support for overlapping structures and/or cognitive resources used in language and music (Besson and Schon, 2001; Slevc et al., 2009; Sammler et al., 2010). In future studies that involve larger groups of children with and without the diagnosis of dyslexia, research may be able to tease apart the behavioral patterns and neural substrates of pitch awareness and phonemic awareness. Determining the extent to which pitch and phoneme processing are connected may help us explore commonalities between tone-deafness and dyslexia, in the hope of designing more refined rehabilitation strategies for dyslexia and perhaps even tone-deafness in the future.

\section{CONCLUSION}

The present results demonstrate an association between phonemic awareness and pitch awareness. Since phonemic awareness is a crucial predictor of reading ability and is disrupted in dyslexia, whereas the agreement between pitch perception and production is characteristic of tone-deaf behavior, the present results suggest that dyslexia and tone-deafness are related and may share a common basis.

\section{ACKNOWLEDGMENTS}

This study was supported by research grants from NIH (R01 DC009823) and Templeton Foundation. We are grateful to all our participants and to Maureen Kroogfor recruiting participants at Grant School \# 7 in Passaic, New Jersey. 


\section{REFERENCES}

Anvari, S. H., Trainor, L. J., Woodside, J., and Levy, B. A. (2002). Relations among musical skills, phonological processing, and early reading ability in preschool children. J. Exp. Child. Psychol. 83, 111-130.

Benasich, A. A., Choudhury, N., Friedman, J. T., Realpe-Bonilla, T., Chojnowska, C., and Gou, Z. (2006). The infant as a prelinguistic model for language learning impairments: predicting from event-related potentials to behavior. Neuropsychologia 44, 396-411.

Besson, M., and Schon, D. (2001). Comparison between language and music. Ann. N. Y. Acad. Sci. 930, 232-258.

Bolduc, J., and Montésinos-Gelet, I. (2005). Pitch processing and phonological awareness. Psychomusicology 19, 3-14.

Bradley, L., and Bryant, P. (1985). Rhyme and Reason in Reading and Spelling. Ann Arbor: The University of Michigan Press.

Bradley, P., and Bryant, L. (1983). Categorizing sounds and learning to read: a causal connection. Nature 301, 419-421.

Burnett, T. A., and Larson, C. R. (2002). Early pitch-shift response is active in both steady and dynamic voice pitch control. J. Acoust. Soc. Am. 112(3 Pt 1), 1058-1063.

Catani, M., Jones, D. K., and Ffytche, D. H. (2005). Perisylvian language networks of the human brain. Ann. Neurol. 57, $8-16$.

Cuddy, L. L., Balkwill, L. L., Peretz, I., and Holden, R. R. (2005). Musical difficulties are rare: a study of "tone deafness" among university students. Ann. N. Y. Acad. Sci. 1060, 311-324.

Démonet, J.-F., Taylor, M. J., and Chaix, Y. (2004). Developmental dyslexia. Lancet 363, 1451-1460.

Forgeard, M., Schlaug, G., Norton, A., Rosam, C., Iyengar, U., and Winner, E. (2008a). The relation between music and phonological processing in normal-reading children and children with dyslexia. Music Percept. 25, 383-390.

Forgeard, M., Winner, E., Norton, A., and Schlaug, G. (2008b). Practicing a musical instrument in childhood is associated with enhanced verbal ability and nonverbal reasoning. PLoS ONE 3, e3566. doi: 10.1371/journal. pone. 0003566

Foxton, J. M., Dean, J. L., Gee, R., Peretz, I., and Griffiths, T. D. (2004). Characterization of deficits in pitch perception underlying "tone deafness". Brain 127(Pt 4), 801-810.
Gordon, E. E. (1979). Primary measures of musical audiation. Chicago, IL: G.I.A. Publications.

Huss, M., Verney, J. P., Fosker, T., Mead, N., and Goswami, U. (2011). Music, rhythm, rise time perception and developmental dyslexia: perception of musical meter predicts reading and phonology. Cortex 47, 674-689.

Jentschke, S., Koelsch, S., and Friederici, A. D. (2005). Investigating the relationship of music and language in children: influences of musical training and language impairment. Ann. N. Y. Acad. Sci. 1060, 231-242.

Jones, J. L., Lucker, J., Zalewski, C., Brewer, C., and Drayna, D. (2009a). Phonological processing in adults with deficits in musical pitch recognition. J. Commun. Disord. 42, 226-234.

Jones, J. L., Zalewski, C., Brewer, C., Lucker, J., and Drayna, D. (2009b). Widespread auditory deficits in tune deafness. Ear Hear. 30, 63-72.

Kalmus, H., and Fry, D. B. (1980). On tune deafness (dysmelodia): frequency, development, genetics and musical background. Ann. Hum. Genet. 43, 369-382.

Kaufman, A. S., and Kaufman, N. L. (1990). Kaufman BriefIntelligence Test. Circle Pines, MN: AGS Publishing.

Lamb, S. J., and Gregory, A. H. (1993). The relationship between music and reading in beginning readers. Educ. Psychol. 13, 19-27.

Lebrun, M. A., Moreau, P., McNallyGagnon, A., Mignault Goulet, G., and Peretz, I. (in press). Congenital amusia in childhood: a case study. Cortex.

Leppanen, P. H., Richardson, U., Pihko, E., Eklund, K. M., Guttorm, T. K., Aro, M., and Lyytinen, H. (2002). Brain responses to changes in speech sound durations differ between infants with and without familial risk for dyslexia. Dev. Neuropsychol. 22, 407-422.

Liu, F., Patel, A. D., Fourcin, A., and Stewart, L. (2010). Intonation processing in congenital amusia: discrimination, identification and imitation. Brain 133, 1682-1693.

Loui, P., Alsop, D., and Schlaug, G. (2009). Tone-deafness: a disconnection syndrome? J. Neurosci. 29, 10215-10220.

Loui, P., Guenther, F. H., Mathys, C., and Schlaug, G. (2008). Action-perception mismatch in tone-deafness. Curr. Biol. 18, R331-R332.

Mengler, E. D., Hogben, J. H., Michie, P., and Bishop, D. V. (2005). Poor frequency discrimination is related to oral language disorder in children: a psychoacoustic study. Dyslexia 11, 155-173.

Merzenich, M. M., Jenkins, W. M., Johnston, P., Schreiner, C., Miller, S.
L., and Tallal, P. (1996). Temporal processing deficits of language-learning impaired children ameliorated by training. Science 271, 77-81.

Moreno, S., Marques, C., Santos, A., Santos, M., Castro, S. L., and Besson, M. (2009). Musical training influences linguistic abilities in 8 -year-old children: more evidence for brain plasticity. Cereb. Cortex 19 712-723.

Nan, Y., Sun, Y., and Peretz, I. (2010). Congenital amusia in speakers of a tone language: association with lexical tone agnosia. Brain 133, 2635-2642.

Overy, K. (2003). Dyslexia and music. From timing deficits to musical intervention. Ann. N. Y. Acad. Sci. 999, 497-505.

Peretz, I., Brattico, E., Jarvenpaa, M., and Tervaniemi, M. (2009). The amusic brain: in tune, out of key, and unaware. Brain 132, 1277-1286.

Peretz, I., Champod, A. S., and Hyde, K. (2003). Varieties of musical disorders The montreal battery of evaluation of amusia. Ann. N. Y. Acad. Sci. 999, 58-75.

Peynircioglu, Z., Durgunoglu, A., and Oney-Kusefoglu, B. (2002) Phonological awareness and musical aptitude. J. Res. Read. 25, 68-80.

Pfordresher, P. Q. (2005). Auditory feedback in music performance: the role of melodic structure and musical skill. J. Exp. Psychol. Hum. Percept. Perform. 31, 1331-1345.

Rosner, J., and Simon, D. P. (1971). The auditory analysis test: an initial report. J. Learn. Disabil. 4, 384.

Sammler, D., Baird, A., Valabregue, R., Clement, S., Dupont, S., Belin, P., and Samson, S. (2010). The relationship of lyrics and tunes in the processing of unfamiliar songs: a functional magnetic resonance adaptation study. $J$. Neurosci. 30, 3572-3578.

Shaywitz, S. E., and Shaywitz, B. A. (2005). Dyslexia (specific reading disability) Biol. Psychiatry 57, 1301-1309.

Slevc, L. R., Rosenberg, J. C., and Patel, A. D. (2009). Making psycholinguistics musical: self-paced reading time evidence for shared processing of linguistic and musical syntax. Psychon. Bull. Rev. 16, 374-381.

Snow, C. E., Burns, M. S., and Griffin, P. (1998). Preventing Reading Difficulties in Young Children. Washington, DC: National Academy Press.

Stadler, S. (1990). Vocal pitch matching ability in children between four and nine years of age. Eur. J. High Ability 1,33-41.

Stahl, S. A., and Murray, B. A. (1994). Defining phonological awareness and its relationship to early reading. J. Educ. Psychol. 86, 221-234.

Stanovich, K. E. (1988). Explaining the differences between the dyslexic and the garden-variety poor reader: the phonological-core variable-difference model. J. Learn. Disabil. 21, 590-604.

Tallal, P. (2004). Improving language and literacy is a matter of time. Nat. Rev. Neurosci. 5, 721-728.

Tallal, P., and Gaab, N. (2006). Dynamic auditory processing, musical experience and language development. Trends Neurosci. 29, 382-390.

Tallal, P., and Piercy, M. (1973). Developmental aphasia: impaired rate of non-verbal processing as a function of sensory modality. Neuropsychologia 11,389-398.

Vernooij, M.W., Smits, M., Wielopolski, P. A., Houston, G. C., Krestin, G. P., and van der Lugt, A. (2007). Fiber density asymmetry of the arcuate fasciculus in relation to functional hemispheric language lateralization in both rightand left-handed healthy subjects: a combined fMRI and DTI study. Neuroimage 35, 1064-1076.

Wahl, M., Li, Y.-O., Ng, J., LaHue, S. C., Cooper, S. R., Sherr, E. H., and Mukherjee,P. (2010). Microstructural correlations of white matter tracts in the human brain. Neuroimage 51, 531-541.

Wood, F. B., Hill, D. F., Meyer, M. S. and Flowers, D. L. (2005). Predictive assessment of reading. Ann. Dyslexia 55, 193-216.

Conflict of Interest Statement: The authors declare that the research was conducted in the absence of any commercial or financial relationships that could be construed as a potential conflict of interest.

Received: 16 February 2011; accepted: 13 May 2011; published online: 30 May 2011. Citation: Loui P, Kroog K, Zuk J, Winner $E$ and Schlaug G (2011) Relating pitch awareness to phonemic awareness in children: implications for tone-deafness and dyslexia. Front. Psychology 2:111. doi 10.3389/fpsyg.2011.00111

This article was submitted to Frontiers in Auditory Cognitive Neuroscience, a specialty of Frontiers in Psychology. Copyright $\odot 2011$ Loui, Kroog, Zuk, Winner and Schlaug. This is an open-access article subject to a non-exclusive license between the authors and Frontiers Media SA, which permits use, distribution and reproduction in other forums, provided the original authors and source are credited and other Frontiers conditions are complied with. 